\title{
CLINICAL PROFILE AND OUTCOME OF NEONATES ADMITTED TO A SECONDARY-LEVEL NEONATAL INTENSIVE CARE UNIT IN NORTH INDIA
}

\author{
SHAGUFTA YOUSUF ${ }^{1}$, SHOWKAT HUSSAIN TALI ${ }^{2 *}$, IFTIKHAR HUSSAIN ${ }^{3}$
}

${ }^{1}$ Department of Obstetrics and Gynaecology, Adesh Institute of Medical Sciences, Bathinda, Punjab, India. ${ }^{2}$ Department of Pediatrics, Adesh Institute of Medical Sciences, Bathinda, Punjab, India. ${ }^{3}$ Department of Social Preventive Medicine, Government Medical College Srinagar, Jammu and Kashmir, India. Email: drshowkatshifa@gmail.com.

Received: 09 May 2017, Revised and Accepted: 06 June 2017

ABSTRACT

Objectives: The objective of this study was to evaluate the performance of a secondary-level neonatal intensive care unit (NICU).

Methods: A total of 336 neonates were enrolled in the study. Their clinical profiles at admission and final outcomes were recorded in a predesigned pro forma.

Results: Hyperbilirubinemia, sepsis, and perinatal asphyxia were the most common reasons for admission. Eighty-five percentage of the neonates could be managed in secondary-level newborn unit with comparable mortality and mortality to a tertiary-level newborn care unit.

Conclusion: Strengthening of secondary newborn care units is a viable option that will help to decrease the burden of tertiary-level NICUs.

Keywords: Neonate, Clinical profile, Outcome, Secondary-level neonatal intensive care unit.

(C) 2017 The Authors. Published by Innovare Academic Sciences Pvt Ltd. This is an open access article under the CC BY license (http://creativecommons. org/licenses/by/4. 0/) DOI: http://dx.doi.org/10.22159/ajpcr.2017.v10i9.19799

\section{INTRODUCTION}

As of 2015 , in India, the neonatal mortality rate is $28 / 1000$ live births [1]. The 2030 Agenda for Sustainable development (WHO) calls for reduction in neonatal mortality to $12 / 1000$ live births by 2030 [2]. Most term neonates can be managed at home under the guidance and supervision of mother or health-care professional. On the other hand, low birth weight and preterm neonates are fragile and vulnerable and they demand high level of skills and technology in a special care nursery or neonatal intensive care unit (NICU) for their intact survival. Apart from high mortality, many avoidable disabilities such as cerebral palsy, mental subnormality, learning disabilities, and recurrent seizures have their origin in perinatal period $[3,4]$. It is encouraging to note that neonates with a birth weight of $1 \mathrm{~kg}$ had a mortality of $95 \%$ in 1960 and now have 95\% chances of survival. In India where a large number of deliveries are non-institutional, secondary- and communitylevel hospitals form important pillars of health-care delivery. The data from secondary-level newborn care units are sparse, and most of the data on neonatal mortality and morbidity are from tertiarylevel care centers [5-7]. The purpose of this study was to evaluate the performance of a secondary-level hospital and stress the importance of neonatal care at this level.

\section{METHODS}

This study was carried out at LD Hospital which is an associated hospital of Government Medical College Srinagar and the main maternity care hospital in the state of Jammu and Kashmir. The hospital has a neonatal care unit attached to it that can be compared to a secondary-level NICU. At the time of study, it was a 25-bedded nursery cum NICU with 15 radiant warmers, 3 phototherapy units, 15 infant trolleys, 4 ventilators, and central oxygen supply. However, these ventilators were less often used due to lack of adequately trained personnel present round the clock. One consultant (pediatrician), one senior resident (MD/DCH Pediatrics), 4 junior residents (MBBS), 4 nurses, and two nursing orderlies would be present during the day duty. One postgraduate student, 2 junior residents, 3 nurses, and 2 nursing orderlies would be present during the night shifts. A consult would be on call round the clock. There were round-the-clock facilities for emergency X-ray and basic laboratory investigations. The study was commenced after obtaining clearance from hospital's ethical committee and written informed consent from one of the parents or the guardian.

\section{Guidelines followed in the NICU}

1. Only inborn neonates were admitted as per hospital policy.

2. Preterm neonates $<32$ weeks, birth weight $<1200$ g, preterm with severe respiratory distress syndrome, stage 2 and 3 hypoxic-ischemic encephalopathy, cyanotic congenital heart diseases, and other serious congenital malformations were referred to higher centers whenever possible.

3. Neonates between $1200 \mathrm{~g}$ and $1500 \mathrm{~g}$ and stable were managed as per standardized protocol. Unstable neonates were referred to higher centers.

Table 1: Demography and other baseline characteristics

\begin{tabular}{ll}
\hline Attribute & $\mathbf{n}(\%)$ \\
\hline Sex & \\
$\quad$ Male & $200(59.5)$ \\
$\quad$ Female & $136(40.5)$ \\
Residence & \\
$\quad$ Rural & $202(60.1)$ \\
$\quad$ Urban & $92(39.9)$ \\
Place of delivery & \\
$\quad$ Home delivery & 0 \\
$\quad$ Hospital delivery & $336(100)$ \\
Birth weight & \\
AGA & $220(65.5)$ \\
SGA & $116(35.5)$ \\
Gestational age (weeks) & \\
$\quad<32$ & $8(2.4)$ \\
32-34 & $88(26.2)$ \\
$>34$ & $240(71.4)$ \\
\hline
\end{tabular}

AGA: Appropriate for gestational age, SGA: Small for gestational age 
Table 2: Clinical profile and outcome of neonates $(n=336)$

\begin{tabular}{|c|c|c|c|}
\hline \multirow[t]{2}{*}{ Attribute } & \multicolumn{3}{|l|}{ n (\%) } \\
\hline & Survived & Expired & Transferred to higher center \\
\hline \multicolumn{4}{|l|}{ Preterm $(<37$ weeks) $(n=96)$} \\
\hline Preterm with hyaline membrane disease & $2(0.6)$ & $10(3)$ & $24(7.1)$ \\
\hline Sepsis & $14(4.1)$ & $10(3)$ & \\
\hline Other morbidities & $32(9.5)$ & $4(1.2)$ & \\
\hline \multicolumn{4}{|l|}{ Term $(>37$ weeks) $(\mathrm{n}=240)$} \\
\hline Perinatal asphyxia & $50(14.8)$ & $11(3.3)$ & $24(7.1)$ \\
\hline Sepsis & $32(9.5)$ & $7(2)$ & \\
\hline Pneumonia & $12(3.5)$ & $1(0.3)$ & \\
\hline Hyperbilirubinemia & $78(23.2)$ & $-\operatorname{los}^{-}$ & \\
\hline Congenital heart disease & $2(0.6)$ & $1(0.3)$ & \\
\hline Hypoglycemia & $6(2.8)$ & - & \\
\hline Other morbidities & $16(4.7)$ & - $\quad$ & \\
\hline Total $(n=336)$ & $244(72.6)$ & $44(13.1)$ & $48(14.3)$ \\
\hline \multicolumn{4}{|l|}{ Geography $(n=336)$} \\
\hline Urban & $77(22.9)$ & $15(4.5)$ & $13(3.9)$ \\
\hline Rural & $167(49.7)$ & $29(8.6)$ & $35(10.4)$ \\
\hline
\end{tabular}

4. Stable neonates more than $1500 \mathrm{~g}$ and 34 weeks were generally kept with mother if there was no associated morbidity or high risk for neonatal sepsis. They were started on breast feeds or expressed breast milk by katori/spoon under medical supervision and were monitored for feeding difficulty, respiratory distress, jaundice, and cyanosis for 48-72 hrs. Neonates who would behave abnormally would be shifted to the NICU.

\section{RESULTS}

Demographic and other baseline characteristics are shown in Table 1. Clinical profile and outcome of neonates are shown in Table 2.

\section{DISCUSSION}

A total of 336 neonates were admitted during the study period. Ninety-six $(28.6 \%)$ neonates were early preterm $(<34$ weeks $)$ and $240(71.4 \%)$ were late preterm and term. Out of these, $244(72.6 \%)$ survived, $44(13.1 \%)$ died, and $48(14.3 \%)$ were transferred to higher centers. As many as $35.5 \%$ of neonates were small for gestational age. Hyperbilirubinemia (23.2), perinatal asphyxia (14.8), and sepsis (9.5) were the major reasons for admission. Shah et al. [8] reported in their study that the share of preterm in neonatal admission was as high as 24 (Table 1). Modi and Kirubakaran [9] reported suspected sepsis (23.7\%), low Apgar score (5.3\%), preterm delivery (14.2\%), and jaundice $(13.4 \%)$ as the major reasons for admission.

Our study highlights that as many as $85 \%$ of neonates can be managed at secondary level with a survival of $72.6 \%$ (Table 2). This is in conformity with the study conducted by Garg et al. [10]. In their study, the overall survival rate was $65 \%$. Forty-two $(25 \%)$ neonates expired and $5.4 \%$ were referred to higher centers. Shah et al. [8] reported an overall mortality of $20.2 \%$ during hospital stay. Bose et al. [11] also reported similar findings. Of the 175 neonates, 6 expired, 2 were discharged against medical advice, and 8 were transferred to the nursery in the tertiary hospital.

There was an appreciable difference in urban and rural mortality rates (Table 1). Mortality in rural population was 2 times than that of urban population. This may be due to poor antenatal care of rural women. This is in conformity with the National Family Health Survey 3 [12].

Mortality and morbidity profile was similar to those reported from tertiary care level centers. Modi and Kirubakaran [9] observed that all the neonates admitted to tertiary care level neonatal unit at CMC Vellore, there were 87 early neonatal deaths, 4\% (49) of inborn admissions and 18\% (38) of out born admissions. Basnet and Shrestha [13] in their study at a tertiary care level hospital also observed neonatal mortality ranges from 9.46 to 14.88 per 1000 live births per year. Ahmed et al. [14] observed a mortality of $17 \%$.

\section{CONCLUSION}

Strengthening of secondary level newborn care units is a viable option that will help to decrease the burden of tertiary level NICUs.

\section{REFERENCES}

1. Available from: http://www.unicef.org/infobycountry/indiastatistics. html. [Last accessed on $2017 \mathrm{Apr}$ 04].

2. United Nations Transforming our world: The 2030 Agenda for Sustainable Development. New York, NY, USA: United Nations; 2015. Available from: http://www.tinyurl.com/od9mens. [Last accessed on 2017 Apr 04].

3. Singh M. Care of New the born. $7^{\text {th }}$ ed. New Delhi: CBS Publishers \& Distributors; 2010

4. Choure MK, Jadhav RR, Padwal SL. Drug utilization study in neonatal intensive care unit at rural tertiary care hospital. Asian J Pharm Clin Res 2017:10(4):102-4

5. National Neonatology Forum. Report of the National-Perinatal Database 2000, New Delhi; 2001.

6. Singh M, Deorari AK, Khajuria RC, Paul VK. A four year study on neonatal morbidity in a New Delhi hospital. Indian J Med Res 1991;94:186-92.

7. Chavan YS, Dattal MS, Khadilkar VV. Causes of early neonatal mortality. Indian Pediatr 1992;29:781-3.

8. Shah GS, Yadav S, Thapa A, Shah L. Clinical profile and outcome of neonates admitted to neonatal intensive care unit (NICU) at a tertiary care centre in Eastern Nepal. Paediatr Soc 2013;33(3). DOI: 10.3126/ jnps.v33i3.8447.

9. Modi N, Kirubakaran C. Reasons for admission, causes of death and costs of admission to a tertiary referral neonatal unit in India. J Trop Pediatr 1995;41(2):99-102.

10. Garg P, Krishak R, Shukla DK. NICU in a community level hospital. Indian J Pediatr 2005;72(1):27-30

11. Bose A, Sinha S, Choudhary N, Aruldas K, Moses PD, Joseph A. Experiences of neonatal care in a secondary level hospital. Indian Pediatr 1999:36(8):802-6.

12. National Family Health Survey 3 (NFHS 3) 2005-2006. International Institute for Population Sciences, Mumbai; India, ORC Macro, Maryland. Available from: http://www.rchiips.org/ARCH-3.htm. [Last accessed on 2017 May 06].

13. Basnet S, Shrestha L. Trends in neonatal mortality at a tertiary level teaching hospital. J Nepal Pediatr Soc 2013;33(3). DOI: 10.3126/jnps. v33i3.8957.

14. Ahmed A, Lutfi S, Al-Hail M, Al-Saadi M. Antibiotic susceptibility patterns of microbial isolates from blood culture in the neonatal intensive care unit of Hamad medical corporation (HMC), Doha, Qatar. Asian J Pharm Clin Res 2013;6(2):191-5. 